\title{
Co-Evolution of Supermassive Black Holes and Their Host Galaxies
}

\author{
J. K. Kotilainen ${ }^{1}$, R. Decarli ${ }^{2}$, R. Falomo ${ }^{3}$, A. Treves ${ }^{4}$, M. Labita ${ }^{4}$, \\ and R. Scarpa ${ }^{5}$ \\ ${ }^{1}$ Finnish Centre for Astronomy with ESO (FINCA) and Tuorla Observatory, University of \\ Turku, FI-21500 Piikkiö, Finland \\ Email: jarkot@utu.fi \\ ${ }^{2}$ Max-Planck-Institut für Astronomie, Königstuhl 17, D-69117 Heidelberg, Germany \\ ${ }^{3}$ INAF - Osservatorio Astronomico di Padova, Vicolo dell'Osservatorio 5, I-35122 Padova, Italy \\ ${ }^{4}$ Università dell'Insubria, via Valleggio 11, I-22100 Como, Italy \\ ${ }^{5}$ Instituto de Astrofisica de Canarias, C/ Via Lactea, s/n E38205 - La Laguna, Tenerife, Spain
}

\begin{abstract}
We study the evolution of the $M_{\mathrm{BH}} / M_{\text {host }}$ relation up to $z=3$ for a sample of 96 quasars with known host galaxy luminosities. Black hole masses are estimated assuming virial equilibrium in the broad-line regions, while the host galaxy masses are inferred from their luminosities. With this data, we are able to pin down the evolution of the $M_{\mathrm{BH}} / M_{\text {host }}$ relation over $85 \%$ of the age of the universe. While the $M_{\mathrm{BH}} / L_{\text {host }}$ relation remains nearly unchanged, taking into account the aging of the stellar population, we find that the $M_{\mathrm{BH}} / M_{\text {host }}$ ratio $(\Gamma)$ increases by a factor $\sim 7$ from $z=0$ to $z=3$. We show that the evolution of $\Gamma$ is independent of radio loudness and quasar luminosity. We propose that the most massive black holes, in their quasar phase at high-redshift, become extremely rare objects in host galaxies of similar mass in the local universe.
\end{abstract}

Keywords. galaxies: active, galaxies: evolution, galaxies: high-redshift, galaxies: nuclei (galaxies:) quasars: general

\section{Introduction}

Many pieces of evidence suggest that supermassive black holes (BHs) and their host galaxies share a joint evolution: (1) the evolution of the quasar luminosity function (LF; Fontanot et al. 2007) closely matches the evolution of the star formation density (Madau et al. 1998), (2) massive BHs are found in virtually all massive galaxies (Decarli et al. 2007), and (3) their masses $M_{\mathrm{BH}}$ are tightly correlated with the stellar velocity dispersion $\sigma_{*}$, luminosity $L_{\mathrm{host}}$, and mass $M_{\text {host }}$ of their host galaxies (e.g., Gultekin et al. 2009).

When and how these relations set in, and which are the physical processes responsible for their onset are still open questions, despite large efforts both theoretically (e.g. Wyithe \& Loeb 2006; Hopkins et al. 2007) and observationally (McLure et al. 2006; Peng et al. 2006; Salviander et al. 2007; Woo et al. 2008).

Probing $\mathrm{BH}$-host galaxy relations at high redshift is extremely challenging. Direct measurements of $M_{\mathrm{BH}}$ from gaseous or stellar dynamics require observations capable of resolving the $\mathrm{BH}$ sphere of influence, which is feasible only for a limited number of nearby galaxies. The $M_{\mathrm{BH}}$ can be measured in galaxies at distances larger than a few tens of Mpc only in type $1 \mathrm{AGN}$, where it can be inferred from the width of emission lines Doppler-broadened by the BH potential well and from the AGN continuum luminosity (e.g., Vestergaard 2002), assuming virial equilibrium. Quasars therefore represent the best tool to probe $M_{\mathrm{BH}}$ at high redshift, thanks to their huge luminosities. Indeed, 
large-field spectroscopic surveys allow estimates of $M_{\mathrm{BH}}$ for several thousands of objects (e.g., Shen et al. 2008; Labita et al. 2009a). On the other hand, the nuclei in quasars outshine the emission from the host galaxies, making their detection challenging. Only recently have the intrinsic (e.g., the nucleus-to-host luminosity ratio $N / H$ ) and extrinsic (e.g., the angular size of the host with respect to the angular resolution of the observations) limitations have been overcome, with the current total of $\sim 300$ resolved quasar host galaxies up to $z \sim 3$ (e.g., Kotilainen et al. 2009).

A number of limitations can affect studies of the evolution of the $M_{\mathrm{BH}}-M_{\text {host }}$ relation:

1. All studies to date use different $M_{\mathrm{BH}}$ proxies as a function of redshift (usually based on $\mathrm{H} \beta$ at $z \lesssim 0.5, \mathrm{Mg}$ II for $0.5 \lesssim z \lesssim 2$, and $\mathrm{C}$ IV at $z \gtrsim 1.6$ ).

2. Selection biases related to luminosity or flux limits, range of $N / H$ sampled, and the steepness of the bright end of the galaxy and quasar LFs (e.g., Lauer et al. 2007).

3. As the properties of quasar host galaxies are directly observed only in a limited number of objects, poor statistics usually affect the available datasets. This study represents a significant effort in overcoming all these limitations. For a full discussion, see Decarli et al. (2009a,b).

\section{Evolution of the $M_{\mathrm{BH}}-L_{\text {host }}, M_{\text {host }}$ Relations}

In Figure 1, we compare our $M_{\mathrm{BH}}$ estimates with the predictions of Bettoni et al. (2003) relation, defined by $z \approx 0$ galaxies, and with the expectations of a fixed $M_{\mathrm{BH}} / M_{\mathrm{host}}=$ 0.002 ratio, as observed in local inactive galaxies (Marconi \& Hunt 2003).
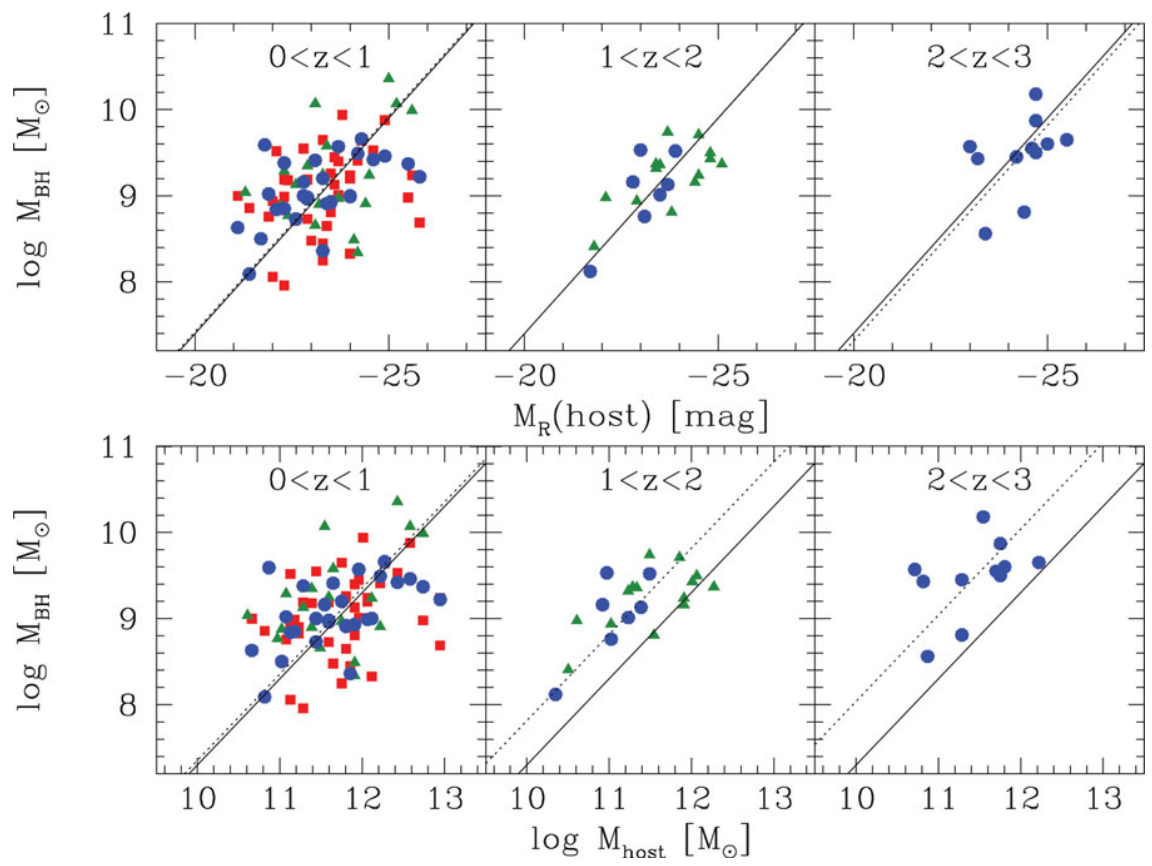

Figure 1. The $M_{\mathrm{BH}}-L_{\text {host }}$ and $M_{\mathrm{BH}}-M_{\text {host }}$ relations in three redshift bins. Squares, triangles and circles mark quasars in which $M_{\mathrm{BH}}$ is derived from $\mathrm{H} \beta, \mathrm{Mg}$ II, and C IV, respectively. The solid line is the Bettoni et al. (2003) relation (upper panels) or $M_{\mathrm{BH}} / M_{\mathrm{host}}=0.002$ (lower panels). The dotted line is the best fit to the data, assuming the slope of the rest-frame relations. Clear evolution is apparent in the $M_{\mathrm{BH}}-M_{\text {host }}$ relationship as a function of redshift. 

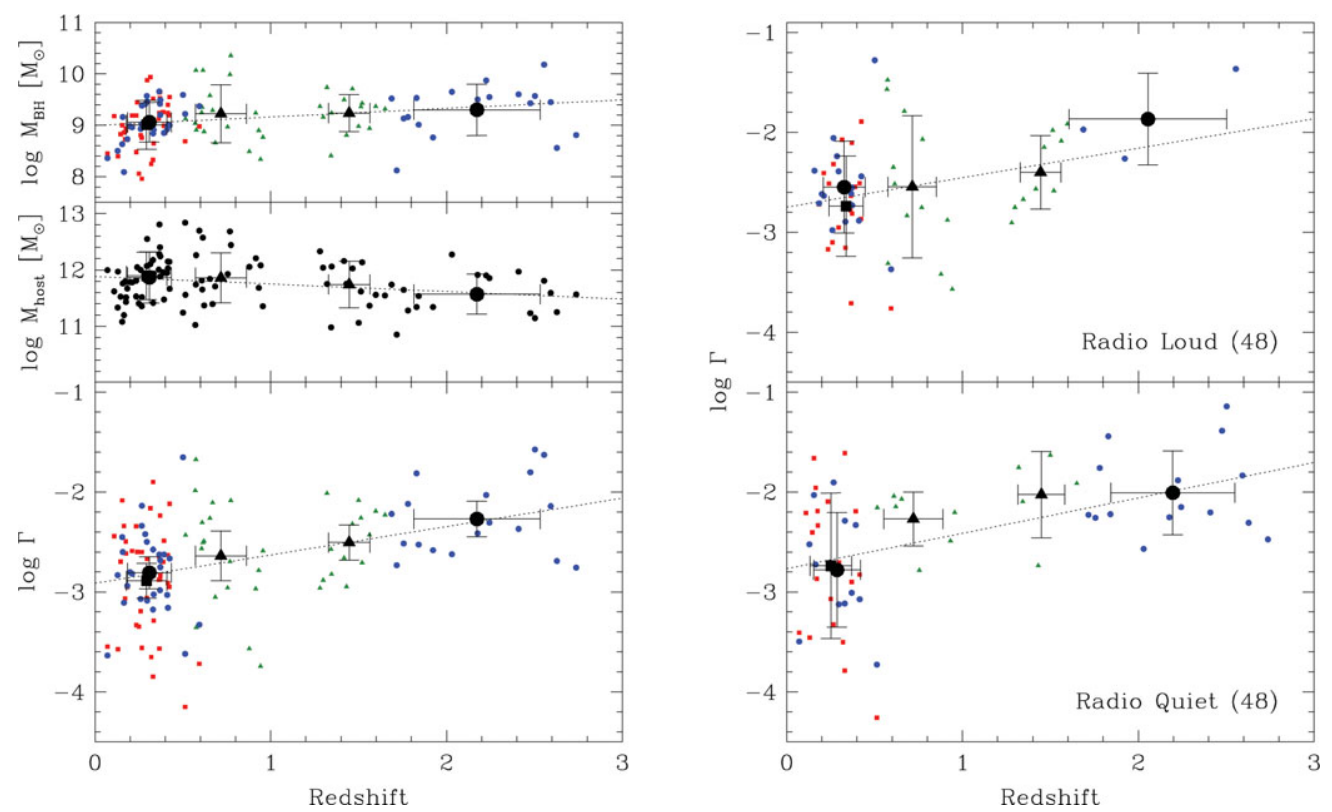

Figure 2. Left: The redshift dependence of $M_{\mathrm{BH}}$ (top panel), $M_{\mathrm{host}}$ (middle panel), and $\Gamma$ (bottom panel). Symbols are as in Figure 1. The best linear fits, and the average points with rms as error bars of the $\mathrm{H} \beta$ (big square), the low- and high- $z$ C IV (big circles) and the $\mathrm{Mg}$ II data with redshift $z<1$ and $z>1$ (big triangles) are shown. Right: The redshift dependence of $\Gamma$ for radio-loud (top panel) and radio-quiet quasars (bottom panel).

The $M_{\mathrm{BH}}-L_{\mathrm{host}}$ relation appears insensitive to cosmic epoch, independent of which line is used to estimate $M_{\mathrm{BH}}$. When correcting for the evolution of the stellar mass-to-light ratio, we find a clear increase $(\sim 0.7 \mathrm{dex})$ of the $M_{\mathrm{BH}} / M_{\text {host }}$ ratio with respect to the local universe. In the left panel of Figure $2, M_{\mathrm{BH}}, M_{\mathrm{host}}$, and their ratio $\Gamma$ are plotted as a function of redshift. The linear best fit of $\log \Gamma$ suggests that galaxies with similar stellar masses harbour BHs $\sim 7$ times more massive at $z=3$ than galaxies at $z=0$.

In the right panel of Figure 2, we compare radio-loud (RLQ) and radio-quiet quasars (RQQ), finding that both are consistent with the $\log \Gamma-z$ relation for the whole sample. The only difference is the offset, in the sense that, at any redshift, both BHs and host galaxies in RLQs are $\sim 0.2$ dex more massive than in RQQs (e.g., Labita et al. 2009b).

\subsection{The Luminosity Function Bias}

Because of the steepness of the bright end of the galaxy luminosity (mass) function and intrinsic scatter in the $M_{\mathrm{BH}}-L_{\text {host }}\left(M_{\text {host }}\right)$ relation, very massive BHs are preferentially found in relatively faint (less massive) galaxies rather than in very rare, extremely bright (massive) galaxies (Lauer et al. 2007). Since high- $z$ samples are dominated by massive objects, this bias increases with $z$, possibly mimicking evolution in $\Gamma$.

To quantify this bias, we assume that $M_{\mathrm{BH}}$ mostly depends on quasar luminosity. This is consistent with the relatively small range of Eddington ratio sampled. If $\sigma_{\mu}$, the cosmic scatter of the $M_{\mathrm{BH}}-L_{\text {host }}$ relation is constant in $L_{\text {host }}$, at a given redshift the bias depends on the shape of the quasar LF. We assume the purely luminosity-evolving LF by Boyle et al. (2000). As a consequence, as long as we sample the same range of the quasar LF at any redshift, the bias is constant. A constant bias is irrelevant for this study, since our 
main aim is to probe the redshift dependence of the $\mathrm{BH}$-host galaxy relations, not their absolute normalization.

The constant luminosity cut at $-26 \mathrm{mag}>M_{V}>-27 \mathrm{mag}$ and the $M_{*}>M_{V}>$ $M_{*}-1$ cut roughly bracket the objects in our sample over five magnitudes in $M_{V}$. Hence, for the whole sample, the bias on $M_{\mathrm{BH}}$ will lie within the expectations from these two cases. We calculate that the bias accounts for $\lesssim 0.11$ dex, i.e., about a factor 1.3 from $z=0$ to $z=3$. As the observed dependence of $\Gamma$ is $\sim 6$ times larger, it cannot be explained by this selection effect.

\subsection{The Effects of the $N / H$ Ratio}

All the quasars in our sample are selected on the basis of their total luminosity, which is dominated by the nuclear light. This introduces a possible bias, where for higher $N / H$, it is more difficult to measure the host-galaxy luminosity, especially at high- $z$. However, the redshift-dependence of $\Gamma$ in high- and low- $N / H$ objects is similar. Moreover, if we include any unresolved quasars, the trend would be even steeper, as they all lie at high- $z$.

\subsection{The Role of Radiation Pressure}

Marconi et al. (2009) suggest that the virial estimates of $M_{\mathrm{BH}}$ may yield lower limits to the true BH mass, if radiation pressure is not taken into account. If the BLR clouds are virialized, a correction can be applied by adding a term depending on the BLR column density $N_{\mathrm{H}}$ and quasar luminosity. However, as the role of the radiation pressure in the BLR is still not clearly understood, especially in the most luminous AGN, we only note that since the average luminosity in our sample increases with $z$, the radiation pressure effect is expected to become more severe at higher $z$, leading to an even steeper increase in $\Gamma$ than that observed.

\section{Discussion}

\subsection{Comparison with Previous Results}

We find that the $M_{\mathrm{BH}} / M_{\text {host }}$ ratio significantly increases with redshift. Here we compare that result with those of other studies in the literature.

McLure et al. (2006) match the average trend of $M_{\mathrm{BH}}$ observed in 38 RLQs at $z<2$ with typical stellar masses of massive radio galaxies at same redshifts. This approach assumes that quasar host galaxies are comparable to massive radio galaxies. Their results may be biased by the different histories of quasar and radio galaxies (e.g., the LFs of AGN evolve differently for various luminosity subclasses). Nevertheless, McLure et al. (2006) find an increase of $\Gamma$ comparable to that observed here. Our results extend these findings to RQQs and beyond the peak age of quasar activity.

Peng et al. (2006) study the evolution of the $M_{\mathrm{BH}}-M_{\text {host }}$ relation in $\sim 20$ low- $z$ quasars and in $\sim 30$ high- $z$ lensed quasars imaged with HST. Their data show a large scatter, possibly due to uncertainties in the modelling of the lens mass distribution and the lens light subtraction. They find practically no evolution in the $M_{\mathrm{BH}}-L_{\mathrm{host}}$ relation, but correcting for the evolution of the stellar population, there is a clear increase in $\Gamma$ at high- $z$ with respect to the local $M_{\mathrm{BH}}-M_{\text {host }}$ relation, in agreement with our findings.

Merloni et al. (2010) study the $M_{\mathrm{BH}}-M_{\text {host }}$ ratio in 89 type 1 AGN with $1<z<$ 2.2 from the zCOSMOS survey. BH masses are derived virially, while the host-galaxy luminosities and stellar masses are inferred from multiwavelength fitting of the SEDs. This technique is effective for intermediate to low-luminosity AGN, though it cannot be applied to quasars, where the nuclear light overwhelms the galaxies. They find that the average $\Gamma$ is higher than observed locally, consistent with the trend observed in our 
study. Jahnke et al. (2009) observed 10 targets with HST and independently derived host galaxy luminosities with a procedure similar to that adopted here (see, e.g., Kotilainen et al. 2009). They find no evolution in the total $M_{\mathrm{BH}}-M_{\mathrm{host}}$ ratio. However, as disks are present in many of their targets, the bulge $M_{\mathrm{BH}}-M_{\text {host }}$ ratio is expected to evolve in agreement with our findings.

Additional indications of the evolution of $\Gamma$ comes from the evolution of $M_{\mathrm{BH}}-\sigma_{*}$ relation. Salviander et al. (2007) use the width of the [O III] narrow emission line as a proxy for $\sigma_{*}$ and study the $M_{\mathrm{BH}}-\sigma_{*}$ relation in $\sim 1600$ quasars up to $z=1.2$ from SDSS. They find that $M_{\mathrm{BH}}$ at high redshift are $\sim 0.2$ dex larger than expected from local $M_{\mathrm{BH}}-$ $\sigma_{*}$ relation. Smaller evolution $(\lesssim 0.1 \mathrm{dex})$ is also proposed by Shen et al. $(2008)$, for 900 type 1 AGN with $z \lesssim 0.4$. Woo et al. (2008) address the $M_{\mathrm{BH}}-\sigma_{*}$ relation in Seyfert galaxies up to $z \sim 0.6$, and find an $M_{\mathrm{BH}}$ excess at high redshift. These findings support our results, notwithstanding the different characteristic luminosities, morphologies, and stellar contents of the sample targets.

These results depict a scenario where, for a given quasar host galaxy, the central BH at high redshift is over-massive with respect to its low- $z$ counterparts. This picture is also consistent with the constraints on the $M_{\mathrm{BH}}-M_{\text {host }}$ evolution derived from the comparison between the galaxy stellar mass function and the quasar LF (Somerville 2009).

\subsection{Why Does $\Gamma$ Evolve?}

If high- $z$ quasars move towards the local $M_{\mathrm{BH}}-M_{\text {host }}$ relation, the unavoidable consequence of our results is that, at a given $M_{\mathrm{BH}}$, galaxy masses increase from $z=3$ to the present age. We sketch three ways for this, and an alternative scenario, where the remnants of high- $z$ quasars keep high $\Gamma$ values down to the present age.

The first scenario involves substantial mass growth of quasar host galaxies through mergers. Strong gravitational interactions may trigger intense gas infall into galaxy centres and lead to activation of $\mathrm{BH}$ accretion. However, $\Lambda$ CDM models (e.g., Volonteri et al. 2003) predict for a massive galaxy only one or two major mergers from $z=3$ to $z=0$, whereas the required increase in the stellar mass of the host galaxies indicates that they would have to suffer three or more major mergers. Furthermore, massive inactive galaxies and quasar host galaxies are likely to have already assembled the majority of their mass at $z \gtrsim 3$ (e.g., Kotilainen et al. 2009). Any episodic rejuvenation of stellar population only marginally affects the mean age of stellar content. If galaxies enter the quasar phase $\sim 1$ Gyr after the activation of the starburst, then the mass involved is $\sim 30 \%$ of the initial mass of the galaxy. Moreover, if quasar host galaxies contain a significant young stellar population, then their $M / L$ ratio would be smaller. Therefore, young host galaxies at high- $z$ would yield even steeper $\Gamma-z$ relation than that observed here.

Another interpretation is that high-redshift quasar host galaxies are gas rich, and form a significant fraction of their stellar content relatively recently. In this picture, the $\mathrm{BH}$ mass is more sensitive to the energy budget or dynamical mass of the galaxy than its stellar mass (e.g., Hopkins et al. 2007). This scenario is disfavoured as all the host galaxies in our sample are massive ellipticals, whose stellar content is usually old. Moreover, if significant recent star formation occurred in quasar host galaxies, the evolution of $\Gamma$ would be much steeper, making this scenario even less realistic.

Inactive, massive ellipticals may be more compact at high redshift than locally (Trujillo et al. 2006). In particular, at high $z$, the velocity dispersion $\sigma_{*}$ is higher. If $M_{\mathrm{BH}}$ regulates the host galaxy $\sigma_{*}$ (e.g., Silk \& Rees 1998) so that the $M_{\mathrm{BH}}-\sigma_{*}$ relation does not evolve significantly, then even a small (a factor $\sim 1.6$ ) increase of $\sigma_{*}$ for a given galaxy would yield a factor of 7 increase in $\Gamma$. However, the average $M_{\mathrm{BH}}$ for a given $\sigma_{*}$ appears to increase from the local to the high- $z$ universe (Salviander et al. 2007; Woo et al. 2008). 
We propose an alternative scenario where the local counterparts of high- $z$ quasars are high-mass outliers above the $M_{\mathrm{BH}}-M_{\text {host }}$ relation. The more massive the $\mathrm{BH}$, the earlier it experiences its quasar phase. These objects are expected to have high $\Gamma$, but they are extremely rare, and contribute only marginally to the present $M_{\mathrm{BH}}-M_{\text {host }}$ relation. In particular, $2<z<3$ quasars should appear locally as inactive massive galaxies with $M_{\mathrm{BH}} \sim 10^{9.5} M_{\odot}$. Assuming the mass function of quasars by Vestergaard \& Osmer (2009), in a volume corresponding to the most distant inactive $\mathrm{BH}$ with a direct mass measurement, virtually no objects are expected with such high values of $\Gamma$.

\section{Conclusions}

We have studied the $M_{\mathrm{BH}}-M_{\text {host }}$ relation as a function of redshift in a sample of 96 quasars from the present age to $z=3$, i.e., over $85 \%$ of the age of the universe. We find that the $M_{\mathrm{BH}}-M_{\text {host }}$ ratio $(\Gamma)$ increases by a factor $\sim 7$ from $z=0$ to $z=3$. This trend is not significantly affected by selection criteria or observational biases. Moreover, it is independent of quasar luminosity and radio loudness. This trend indicates that the most massive black holes, in their quasar phase at high redshift, keep their high values of $\Gamma$ down to the present age, becoming very rare objects in the local universe.

\section{References}

Bettoni, D., Falomo, R., Fasano, G., \& Govoni, F. 2003, A\&A, 399, 869

Boyle, B. J., et al. 2000, MNRAS, 317, 1014

Decarli, R., et al. 2007, MNRAS, 381, 136

Decarli, R., Falomo, R., Treves, A., Kotilainen, J. K., Labita, M., \& Scarpa, R. 2010a, MNRAS, in press [arXiv:0911.2983]

Decarli, R., Falomo, R., Treves, A., Labita, M., Kotilainen, J. K., \& Scarpa, R. 2010b, MNRAS, in press [arXiv:0911.2988]

Fontanot, F., et al. 2007, A\&A A, 461, 39

Gultekin, K., et al. 2009, ApJ, 698, 198

Hopkins, P. F., Hernquist, L., Cox, T. J., Robertson, B., \& Krause, E. 2007, ApJ, 669, 45

Jahnke, K., Wisotzki, L., Courbin, F., \& Letawe, G. 2007, MNRAS, 378, 23

Jahnke, K., et al. 2009, ApJ, 706, L215

Kotilainen, J. K., Falomo, R., Decarli, R., Treves, A., Uslenghi, M., \& Scarpa, R. 2009, ApJ, 703,1663

Labita, M., Decarli, R., Treves, A., \& Falomo, R. 2009a, MNRAS, 396, 1537

Labita, M., Decarli, R., Treves, A., \& Falomo, R. 2009b, MNRAS, 399, 2099

Lauer, T. R., Tremaine, S., Richstone, D., \& Faber, S. M. 2007, ApJ, 670, 249

Madau, P., Pozzetti, L., \& Dickinson, M. 1998, ApJ, 498, 106

Marconi, A. \& Hunt, L. K. 2003, ApJ, 589, L21

Marconi, A., et al. 2009, ApJ, 698, L103

McLure, R. J., Jarvis, M. J., Targett, T. A., Dunlop, J. S., \& Best, P. N. 2006, MNRAS, 368, 1395

Merloni, A., et al. 2010, ApJ, 708, 137

Peng, C. Y., et al. 2006b, ApJ, 649, 616

Salviander, S., Shields, G. A., Gebhardt, K., \& Bonning, E. W. 2007, ApJ, 662, 131

Shen, J., Vanden Berk, D. E., Schneider, D. P., \& Hall, P. B. 2008b, AJ, 135, 928

Silk, J. \& Rees, M. J. 1998, A\&SA, 331, L1

Somerville, R. 2009, MNRAS, 399, 1988

Trujillo, I., et al. 2006, ApJ, 650, 18

Vestergaard, M. \& Osmer, P. S. 2009, ApJ, 699, 800

Volonteri, M., Haardt, F., \& Madau, P. 2003, ApJ, 582, 559

Woo, J.-H., Treu, T., Malkan, M. A., \& Blandford, R. D. 2008, ApJ, 681, 925

Wyithe, J. S. B. \& Loeb, A. 2006, ApJ, 634, 910 